\title{
ATM Transaction Security System Using Biometric Palm Print Recognition
}

\author{
Janani.V, L. Hanapraveena \\ (Department Of ECE/ Jeppiaar Engineering College, Chennai) \\ (Department Of ECE/ Jeppiaar Engineering College, Chennai)
}

\begin{abstract}
Biometrics-based authentication offers several advantages over other authentication methods, there has been a significant surge in the use of biometrics for user authentication in recent years. The software be designed will control a simulated automated teller machine (ATM) with a customer console for interaction with the customer. The ATM will communicate with the bank computer over an appropriate communication link. The ATM will service one customer at a time. A customer will be required to enter a login id and validate his finger print and both will be sent to the bank for validation as part of each transaction. This makes the developed ATM software more secure as compared to the software that authenticates the user merely by using a PIN or password. The end result is an enhanced biometric authenticated ATM system that ensures greater security and increased customer's confidence in the banking sector.
\end{abstract}

Keywords: palm-print recognition, PIN, biometric authentication, security.

\section{Introduction}

This paper proposes a system which takes the user biometric palm print compares it with the image stored in database on a server and provides more secure financial transaction. Nowadays various electronic devices use biometrics for fast identification and authentication. Computer systems in offices, banks uses different pins for transaction for secured reasons. General methods of identification using ID cards and pin is not reliable. An embedded palm print biometric authentication scheme for automated teller machine (ATM) banking systems is proposed in this project. In this scheme, a palm print biometric technique is fused with the ATM for person authentication to improve the security level.Nowadays a lot of criminals activities occur in banking services. Criminals interfere with the ATM terminal and steal customers card details by illegal means .Once user bank card is lost and the password is stolen, the user account is vulnerable to attack.Traditional ATM systems authenticate generally by using a card (credit, debit, or smart) and a pass word or PIN. This PIN has some defects. The prevailing techniques of user authentication, which involves the use of either passwords and user IDs (identifiers), or identification cards and PINs (personal identification numbers), suffer from several limitations. Passwords and PINs can be illicitly acquired by direct secret observation. When credit and ATM cards are lost or stolen, an unauthorized user can often come up with the correct personal codes. Despite warning, many people continue to choose easily guessed PINs and passwords - birthdays, phone numbers and social security numbers. Biometric authentication technology may solve this problem since a person's biometric data is undeniably connected to its owner, is nontransferable and unique for every individual. The system can compare scans to records stored in a central or local database or even on a smart card. Biometrics can be defined as a measurable physiological and behavioral characteristic that can be captured and subsequently compared with another instance at the time of verification. It is automated methods of recognizing a person based on a physiological or behavioral characteristic. The palm print has maximum region of interest; which makes researcher to study and carry experiments over palm print image such as palm print features - ridge, minutia and principal lines etc. the proposed work discusses about the person identification on the basis of physiological characteristics of human being that is palm print recognition. The principal lines of the palm print are extracted . once these principal lines extracted from the image will be feature for the person identification. Then we have calculated the basic statistical properties of the palm print image; hence these calculated values are found to be distinct and unique for the number of experimented palm print image sample.

\section{Literature Survey}

Palm print recognition has been investigated over the past ten years. During this Period, many different problems related to palm print recognition have been addressed. Researchers have focused on developing accurate verification algorithms. Various feature extraction and matching algorithms have been proposed. An automated palm print recognition system evaluated the results in terms of correct recognition rate and verification rate. To achieve high verification accuracy, researchers combine different biometric traits with palm prints and combine different features in palm prints. Researchers also address a more challenging problem, realtime palm print identification in large database.In this context, both accuracy and recognition speed are 
important recently, the biometric community has also emphasized on the security of biometric systems. Pioneers have proposed some measures to protect palm print systems In addition to summarizing the current palm print research; other related issues like privacy involved with palm prints are discussed. There are various biometrics system, but they have failure rate in high proportion. Some of them are mentioned below:-

\section{1) Face Recognition:-}

Face recognition is a Biometric technology that uses an image either from a camera or photograph to recognize a person. It does not requires a person's cooperation. Face recognition is completely oblivious to differences in appearance as a result of race or gender differences and is a highly robust Biometrics. However, the face changes considerably with age, and even due to make-up and expression changes. Face recognition systems can be divided into two main categories. Systems used to verify the identity of a person in a known environment at a fairly constant distance and systems that try to identify a person from a group of people in a dynamic environment and at a random distance.

\section{2) Iris Recognition:-}

The iris at first seems to be a bad choice for a biometric. But if observed closely, it has considerable texture detail that makes it a good biometric trait. Iris recognition is not considered to be safe. Iris recognition technology is also not so accurate and it can be fooled.

\section{3) Ear Recognition:-}

Ear is a relatively new class of biometrics. The shape and size of each ear varies from person to person which makes it a trait. Several approaches such as two-stage scale and rotation invariant geometric approach which is based on the concept of max-line, the longest line that has both its end points on the edges of the ear, have been proposed for ear recognition. But also according to research it had shown most failure.

reports.

\section{4) Voice Recognition:-}

Voice recognition systems work by analyzing the waveforms and air pressure patterns produced while a person talks. These systems may use the characteristics of an individual voice or some pre-arranges words. Voice is one of the most convenient biometric but is not reliable due to bad accuracy. Voice can be mimicked and also a person with a cold or throat problems may face problems using the voice recognition system as it may be rejected.

\section{Implementation Details}

The palm-print recognition system includes preprocessing followed by ROI extraction. After ROI extraction, features are extracted using the feature extraction algorithms. Then matching is done on the basis of the extracted features. The palm-print is then accepted or rejected. The proposed palmprint recognition system has been depicted below:

Flowchart of palm-print recognition is shown below:

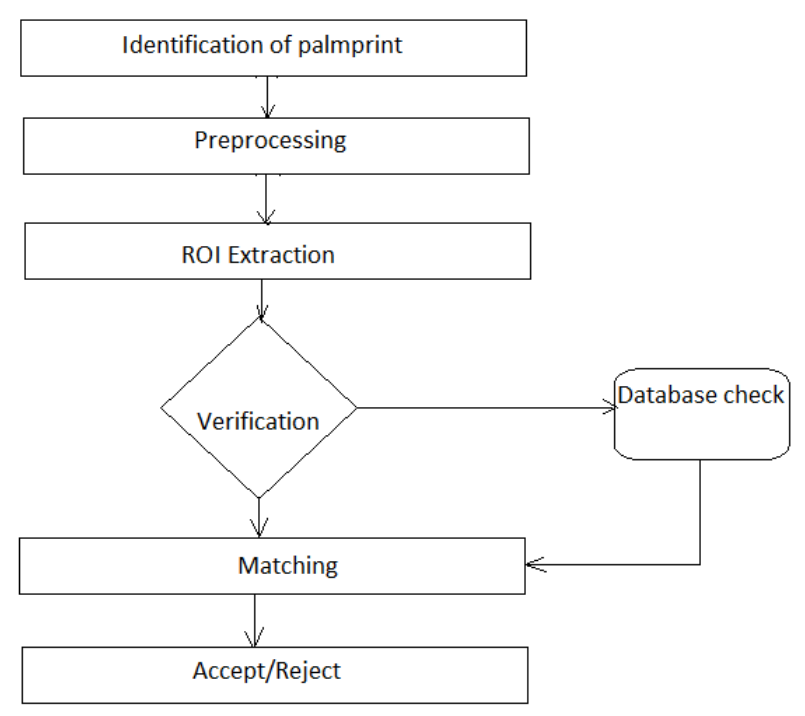




\section{A. Image Acquisition \& Alignment}

Our image acquisition setup is inherently simple and does not employ any special illumination. A palm scanner was used to acquire the hand images. The users were only requested to make sure that

(i) Their fingers do not touch each other and

(ii) Most of their hand (back side) touches the imaging table.

\section{B. Preprocessing}

To reduce the overhead, instead of directly using the palmprint images, preprocessing needs to be done. Preprocessing is used to remove distortion, align the palm-prints and to crop the region of interest. This cropped ROI is used for feature extraction. This is done in some steps:

a. The palm image is binarized.

b.Boundary tracking method is implemented.

c. Detection of key points.

d. Coordination system is established.

e. Extracting the central part.

\section{ROI Extraction}

Preprocessing is done and after that the central part is segmented. There are some algorithms like segment circular, half elliptical or square regions for feature extraction. Here the square region is easiest and widely used. The cropped image is taken and the minor lines get suppressed by passing through low pass filter.

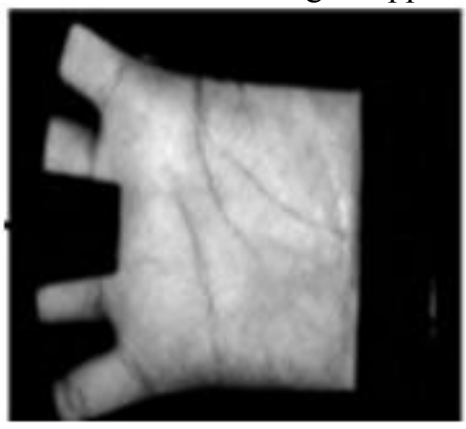

The Original Image

We will get the following cropped image from original one.

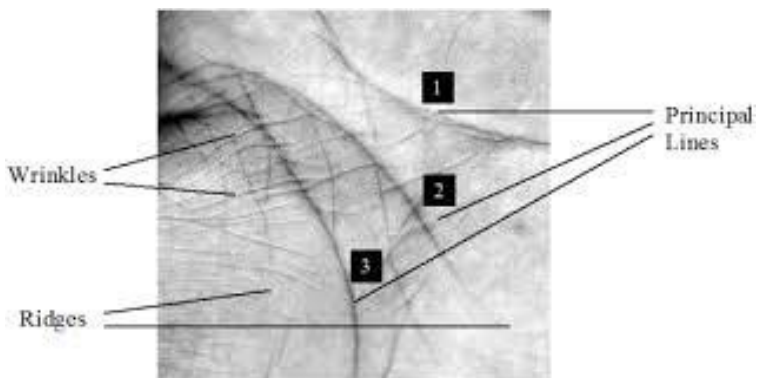

The Cropped Image

A square structuring element (SE) is used to probe the composite binarized image. The center of binary hand image after erosion, i.e., the center of rectangle that can enclose the residue is determined. This center coordinates are used to extract a square palm-print region of fixed size as shown in figure .

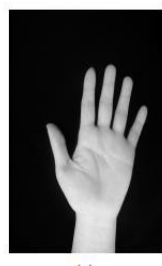

(a)

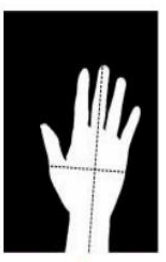

(b)

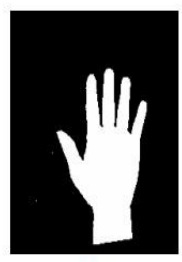

(c)

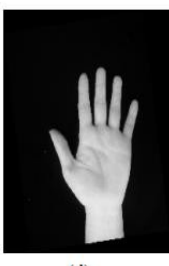

(d)

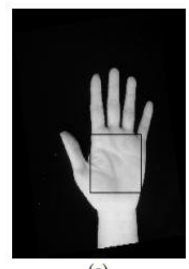

(e)

\section{Feature Extraction}

The palmprint pattern is mainly made up of palm lines, i.e., principal lines and creases. Line feature matching is reported to be powerful and offers high accuracy in palmprint verification. However, it is very 
difficult to accurately characterize these palm lines, i.e. ,their magnitude and direction, in noisy images. Therefore, a robust but simple method is used here and the approaches are follows:

Line based approaches: This approach develops edge detectors and makes use of the magnitude of the palm lines. The magnitudes of the palm lines are projected in $\mathrm{x}$ and $\mathrm{y}$ coordinates forming histograms. After this, the first and second order derivatives of the palm images are calculated.The first order derivative is used to identify the edge points and corresponding directions. The second order derivative is used to identify the magnitude of lines. Then the Euclidian distance is used for matching.

Subspace Based Approaches: Sub space based method is also called appearance based approach, generally involve principal component analysis (PCA), Linear discriminant analysis (LDA) and independent component analysis (ICA). The subspace coefficient are considered as features .In addition to applying PCA, LDA and ICA directly to palm print images, researchers also employ wavelets, Discrete cosine transform and kernel in their method.

Statistical Approaches: There are two types local and global. The local approaches transform images into another domain and divide the transform into several regions such as mean and variance of each small region. Researchers compute global statistical features like moments, centre of gravity and density directly from the whole transformed images. Yong et al. method for feature extract ion divides the palm print image into a set of $\mathrm{n}$ small regions and then calculates the mean and S.D of sub regions. Euclidian square norm is employed for matching.

Coding Approaches: Palm code uses a single Gabor filter to extract the local phase information of palm print. Kong et al. introduced a fusion code method to encode the phase of the filter responses from a bank of Gabor filters with different orientations. A practical palmprint recognition algorithm using $2 \mathrm{D}$ phase information

(a) reduces the registered data size by registering quantized phase information and

(b) deals with nonlinear distortion between palmprint images by local block matching using Phase-Only Correlation.

\section{E. Matching and Database Verification}

After the feature extraction the users are authenticated by palmprint recognition systems. The image is matched with the image stored in database server whether it is present there or not. This matching is done on the basis of extracted features. This classification is used for the palm images to be accepted or not. Also it is verified with the stored image in the server. If the user's Image verifies with the actual image stored in server then transaction is allowed else rejected.

In case of emergency situations if your card is accessed by your neighbour,the second option is available where the user can enter the PIN number and click second user then automatically the message will be send to the card holder with the duplicate password.The Card holder will inform the password to the second user and the transaction will be completed.

\section{Module Implementation}

No matter how you convert your images, it is a good idea to print the files to make sure nothing was lost in the procedure. The modules of the project are given below:-

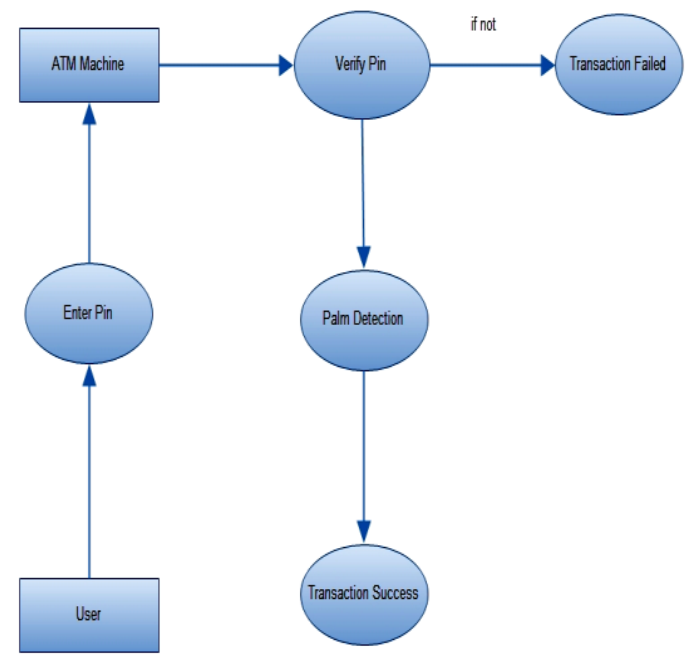

Architectural Block Diagram 


\section{A. Bank}

This will be a bank server which have following operations:-

1) Add new user

2)Deposit money

3)Withdraw money

4)Balance enquiry

5)View/update account details

\section{B. Account Operations}

It will contain the operations that user want to perform at the bank side these operations are same as admin operations.

\section{ATM Machine}

It will be an user interface from which the user can make transactions with the help of ATM card the various operations Include:-

1) Balance Enquiry

2) Change Pin

3) Withdraw Amount

4) Mini Statement

\section{Bank web Service}

It will use to store and manage user data. It will be responsible for the communication between bank server and ATM machine. The Operations between the different modules stated above are taking place as shown below with the help of sequence diagram.

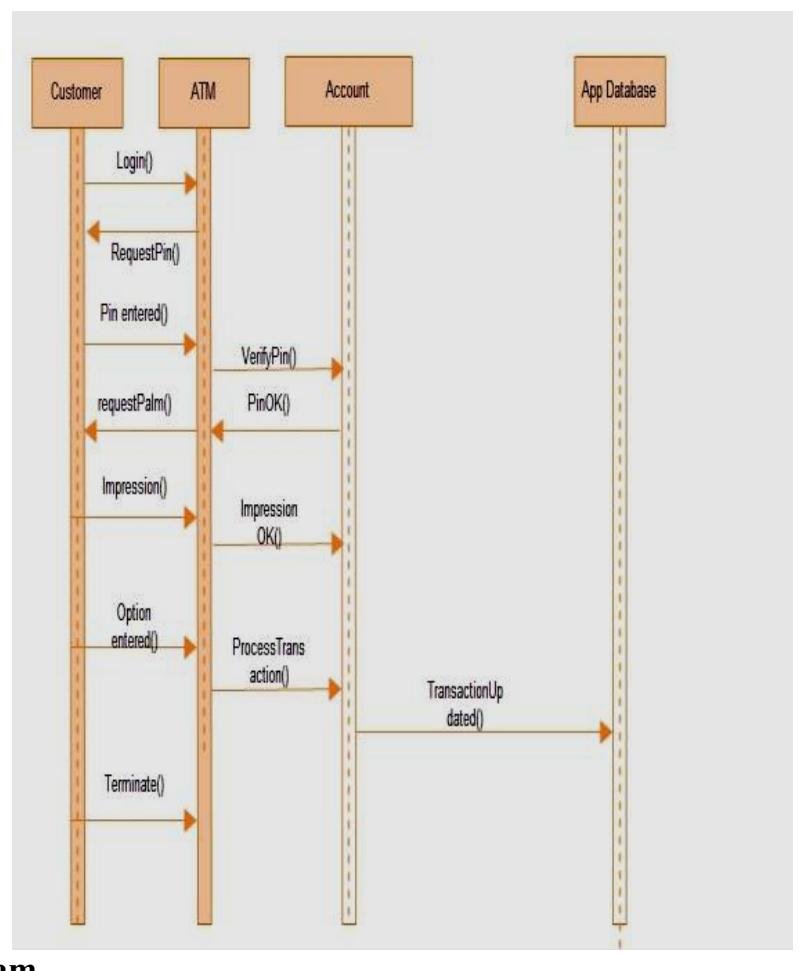

\section{Sequence Diagram}

\section{Conclusion}

Several existing methods are used for ATM security. In the proposed approach, uses pin along with palm-print image at a time, dominant spectral features are been extracted such as the major lines. This approach helps in increasing the performance and accuracy of the system.. The aim of working on the palm-print recognition system is to develop a system with increased speed accuracy and security. 


\section{References}

[1]. Tee Connie, Andrew Teoh Beng Jin, Michael Goh Kah Ong, David Ngo Chek Ling, “An automated palmprint recognition system ”, Image and Vision Computing, Vol.23, pp.501-515, 2005.

[2]. Patprapa Tunkpien, Sasipa Panduwadeethorn, Suphakant Phimoltares, “ Compact Extract ion of Principle Lines in P almprint Using Consecutive Filtering Operations".

[3]. Tee Connie, Andrew Teoh, Michael Goh, David Ngo, "Palmprint Recognition with PCA and ICA" , Image and Vision Computing NZ,Palmerston North, pp.227-232, 2003.

[4]. Jinsu Choi Jaemin Kim, Seongwon Cho and II Robert J. Marks. Iris recognition using wavelet features. Journal of VLSI Signal Processing, 38(2): $147\{156,2004$.

[5]. Hui Chen and Bir Bhanu. Contour matching for $3 \mathrm{~d}$ ear recognition. In Proceedings of the Seventh IEEE Workshops on Application of Computer Vision (WACV/MOTION'05), volume 1, pages $123\{128$, Washington, DC, USA,2005. IEEE Computer Society.

[6]. I Ketut Gede Darma Put ra, Erdiawan, "High Performance Palmprint Identification system Based On Two Dimensional Gabor',TELKOMNIKA Vol. 8, No. 3, pp.309-318, 2010.

[7]. Sina Akbari Mistani, Shervin Minaee, Emad Fatemizadeh, "Multispectral Palmprint Recognition Using a Hybrid Feature" Electrical Engineering Department, Sharif University of Technology, Tehran, pp.1-7, 2011.

[8]. J. H. Connell R. M. Bolle and N. K. Ratha. Biometric perils and patches. Pattern Recognition, 35:2727 \{2738, 2002.

[9]. Shujun Li and Xuan Zheng. On the security of an image encrypt ion In Proceedings of 2002 IEEE International Conference on Image (ICIP 20)

[10]. G. Lu, D. Zhang, K. Wang, Palm print recognition using eigenpalms features, Pattern Recognition Letters 24 (9) (2003). 